\title{
DO THE PHOTOMETRIC COLORS OF TYPE II-P SUPERNOVAE ALLOW ACCURATE DETERMINATION OF
} HOST GALAXY EXTINCTION?*

\author{
Kevin Krisciunas $^{1}$, Mario Hamuy ${ }^{2}$, Nicholas B. SuntzefF ${ }^{1}$, Juan Espinoza ${ }^{3}$, David Gonzalez ${ }^{3}$, Luis Gonzalez ${ }^{2}$, \\ Sergio GonZalez ${ }^{4}$, Kathleen Koviak ${ }^{5}$, Wojtek Krzeminski ${ }^{4}$, Nidia Morrell ${ }^{4}$, Mark M. Phillips ${ }^{4}$, Miguel Roth $^{4}$, AND \\ JoAnna ThOMAS-OSIP \\ ${ }^{1}$ Department of Physics, Texas A\&M University, 4242 TAMU, College Station, TX 77843, USA; krisciunas@physics.tamu.edu, suntzeff@physics.tamu.edu \\ 2 Departmento de Astronomía, Universidad de Chile, Casilla 36D, Santiago, Chile; mhamuy@das.uchile.cl \\ ${ }^{3}$ Cerro Tololo Inter-American Observatory, Casilla 603, La Serena, Chile; jespinoza@ctio.noao.edu \\ ${ }^{4}$ Las Campanas Observatory, Casilla 601, La Serena, Chile; sgonzalez@1co.cl,wojtek@1co.cl, nmorrell@1co.cl, mmp@1coeps1.1co.cl, mroth@1co.cl, jet@1co.cl \\ ${ }^{5}$ Observatories of the Carnegie Institution of Washington, 813 Santa Barbara Street, Pasadena, CA 91101, USA \\ Received 2008 May 26; accepted 2008 September 16; published 2008 December 2
}

\begin{abstract}
We present infrared photometry of supernova (SN) 1999em, plus optical photometry, infrared photometry, and optical spectroscopy of SN 2003hn. Both objects were Type II-P SNe. The $V-[R I J H K]$ color curves of these SNe evolved in a very similar fashion until the end of the plateau phase. This allows us to determine how much more extinction the light of SN 2003hn suffered compared to SN 1999em. Since we have an estimate of the total extinction suffered by SN 1999em from model fits of ground-based and space-based spectra as well as photometry of SN 1999em, we can estimate the total extinction and absolute magnitudes of SN 2003hn with reasonable accuracy. Since the host galaxy of SN 2003hn also produced the Type Ia SN 2001el, we can directly compare the absolute magnitudes of these two SNe of different types.
\end{abstract}

Key words: supernovae: individual (SN 1999em, SN 2003hn) - techniques: photometric

Online-only material: color figures

\section{INTRODUCTION}

Supernovae (SNe) come in two basic models: exploding white dwarfs that are members of close binary systems and single massive stars that develop iron cores.

Type Ia SNe are generally regarded to be exploding carbonoxygen white dwarf stars that have reached the Chandrasekhar limit due to mass transfer from a nearby nondegenerate donor star (Livio 2000, and references therein). Therefore, Type Ia $\mathrm{SNe}$ are explosions constrained by a uniform energy budget. This leads to a high degree of uniformity of their light curves. The objects with more rapidly declining light curves are fainter at maximum light, and the slow decliners are brighter (Phillips 1993; Hamuy et al. 1996; Riess et al. 1996; Perlmutter et al. 1997; Phillips et al. 1999; Jha et al. 2007). In the nearinfrared (near-IR), however, Type Ia SNe are even better-they are standard candles (Krisciunas et al. 2004a, 2004b; WoodVasey et al. 2007). Except for the fastest declining objects, there are essentially no "decline rate relations" in the IR.

As we have shown in a recent series of papers (see Krisciunas et al. 2007; Wang et al. 2008, and references therein), a combination of optical and near-IR photometry allows the accurate determination of host galaxy extinction of Type Ia $\mathrm{SNe}$, even if the dust is different from the "standard" Galactic dust with $R_{V}=3.1$.

Type II SNe are thought to be single stars born with $8 M_{\odot}$ or more, which, after a few million years of evolution, undergo the collapse of their iron cores and the subsequent ejection of their

\footnotetext{
* Based in part on observations taken at the Cerro Tololo Inter-American Observatory, National Optical Astronomy Observatory, which is operated by the Association of Universities for Research in Astronomy, Inc. (AURA) under cooperative agreement with the National Science Foundation. This paper also includes data gathered with the $6.5 \mathrm{~m}$ Magellan Telescopes located at Las Campanas Observatory, Chile.
}

hydrogen-rich envelopes (Heger et al. 2003). Observationally, Type II-P SNe are distinguished by prominent hydrogen lines in their spectra. When the star explodes with a significant fraction of its H-rich envelope, in theory it should display a light curve characterized by a phase of $\sim 100$ days of nearly constant luminosity followed by a sudden drop of 2-3 mag (Litvinova \& Nadezhin 1983). More than half of all Type II SNe belong to this class of "Plateau" SNe whose progenitors are attributed to stars born with less than $25 M_{\odot}$ (Hendry et al. 2006; Li et al. 2007). Type II-P SNe show a wide mass range, a considerable spread in explosive power, absolute magnitudes, and ejecta velocities (Hamuy 2003). Hamuy \& Pinto (2002) have shown that there is a correlation between the expansion velocities of Type II-P $\mathrm{SNe}$ and their bolometric luminosities during the plateau phase. Thus, Type II-P SNe can be useful as standardizable candles in their own right.

In this paper, we address a simple question. Can Type II-P $\mathrm{SNe}$ be found that exhibit similar enough color curves such that we may attribute the systematic differences of their colors to different amounts of dust extinction along the line of sight? If the answer to this question is yes, then in principle photometry can be used to obtain absolute magnitudes of Type II-P SNe, which have minimal systematic errors owing to dust extinction along the line of sight. Accurate distances give us accurate luminosities, which are critical for constraining hydrodynamic models of Type II-P SNe as well as for cosmological studies.

\section{SN $1999 \mathrm{em}$}

SN 1999em was discovered on 1999 October 29.44 by $\mathrm{Li}$ (1999) in the face-on spiral NGC 1637. A spectrum taken on October 30.34 UT by Jha et al. (1999) revealed it to be a Type II SN at an early epoch. Jha et al. (1999) give the correct offsets of the SN from the core of its host galaxy (15.4 arcsec west and 
17.0 arcsec south). This object was intensively followed as part of the Supernova Optical and Infrared Survey (SOIRS). ${ }^{6}$ The Galactic reddening along the line of sight to SN 1999em is small, $E(B-V)=0.040$ (Schlegel et al. 1998), giving $A_{V}(\mathrm{Gal})=$ $0.124 \pm 0.012 \mathrm{mag}$.

A large fraction of the data of the two SNe discussed in this paper were obtained with the dual optical-IR photometer ANDICAM. This instrument was mounted at the Cassegrain focus of the Yale-AURA-Lisbon-Ohio (YALO) $1.0 \mathrm{~m}$ telescope at the Cerro Tololo Inter-American Observatory (CTIO) from 1999 through early 2003. After that it has been used with the $1.3 \mathrm{~m}$ ex-2MASS (Two Micron All Sky Survey) telescope at CTIO.

Optical and near-IR light curves of SN 1999em were first presented graphically by Hamuy et al. (2001) and need not be reproduced here. The optical photometry of SN 1999em will be published by M. Hamuy et al. (2008, in preparation) as part of an extensive study of Type II-P SNe. Leonard et al. (2002) presented independent $U B V R I$ photometry of this object. We note that the data of Leonard et al. were obtained with the Katzman Automatic Imaging Telescope at Lick Observatory, and not with any of the telescopes at CTIO. The photometric colors of SN 1999em as measured with the various telescopes are in very good agreement.

Revised analysis of SN 1999em using the Expanding Photosphere Method (EPM) yields an explosion time of JD 2,451,475 \pm 1 day (M. Jones et al. 2008, in preparation). If all Type II-P $\mathrm{SNe}$ could yield times of explosion with this accuracy, then these would be the most sensible reference times. F. Olivares et al. (2008, in preparation) use the time that signals the end of the plateau. But it is known that the length of the plateau varies, as it depends on the mass of the ejected envelope. For our purposes here we shall adopt a "reference time" of Julian Date 2,451,483.7 (= 1999 November 1.2 UT) for SN 1999em, which corresponds to a date early in the photometric record. The plateau phase of the light curves lasted for roughly 100 days after this time.

The near-IR photometry of SN 1999em is presented in Table 1. The calibration of the IR photometry was accomplished using observations of the SN 1999em field and Persson et al. (1998) standards on five photometric nights.

The $V-[I J H K]$ colors of $\mathrm{SN} 1999 \mathrm{em}$ are shown in Figure 1. These will be our primary references for an investigation of the colors of SN 2003hn. Our color templates were obtained by fitting subsets of SN 1999em data with low-order polynomials, then stitching the fits together. Until 100 days after the "reference time," the rms scatter about these loci is less than \pm 0.02 mag for $V-R$ and $V-I$, and is roughly \pm 0.04 mag for $V-J, V-H$, and $V-K$.

\section{SN $2003 \mathrm{hn}$}

SN 2003hn was discovered on 2003 August 25.7 UT by Evans (2003). The SN was found some 47 arcsec east and 53 arcsec north of the nucleus of the side-on spiral galaxy NGC 1448 (Krisciunas \& Espinoza 2003). This is the same galaxy that hosted the Type Ia SN 2001el (Krisciunas et al. 2003, 2007). Using the $\mathrm{IRAF}^{7}$ tasks ccmap and cctran we find

\footnotetext{
6 The SOIRS project utilized several telescopes in Chile and one in Arizona. See Hamuy (2001) for further details.

7 IRAF is distributed by the National Optical Astronomy Observatories, which is operated by the Association of Universities for Research in Astronomy, Inc., under cooperative agreement with the National Science Foundation.
}

Table 1

Near-IR Photometry of SN 1999em

\begin{tabular}{|c|c|c|c|c|}
\hline $\mathrm{JD}^{\mathrm{a}}$ & $J / J_{s}$ & $H$ & $K / K_{s}$ & Telescope $^{b}$ \\
\hline 481.80 & $13.748(0.015)$ & $13.552(0.015)$ & $13.349(0.015)$ & 1 \\
\hline 482.69 & $13.544(0.032)$ & 13.413 (0.019) & $13.191(0.038)$ & 2 \\
\hline 483.76 & $13.507(0.015)$ & $13.400(0.015)$ & $13.205(0.015)$ & 2 \\
\hline 483.78 & $13.620(0.018)$ & $13.288(0.025)$ & $13.086(0.029)$ & 1 \\
\hline 484.76 & $13.505(0.015)$ & $13.388(0.015)$ & $13.200(0.020)$ & 2 \\
\hline 485.73 & $13.486(0.050)$ & $13.373(0.036)$ & $13.247(0.069)$ & 2 \\
\hline 486.77 & $13.437(0.015)$ & $13.327(0.015)$ & $13.149(0.015)$ & 2 \\
\hline 487.75 & $13.445(0.015)$ & $13.338(0.015)$ & $13.158(0.015)$ & 2 \\
\hline 488.76 & $13.394(0.015)$ & $13.297(0.015)$ & $13.132(0.017)$ & 2 \\
\hline 489.81 & $13.378(0.015)$ & $13.269(0.015)$ & $13.078(0.015)$ & 2 \\
\hline 495.74 & $13.292(0.015)$ & $13.203(0.015)$ & $13.142(0.021)$ & 1 \\
\hline 498.68 & $13.342(0.032)$ & $13.247(0.040)$ & $12.997(0.033)$ & 1 \\
\hline 501.71 & $13.072(0.015)$ & $13.095(0.015)$ & $12.875(0.018)$ & 1 \\
\hline 504.74 & $13.289(0.015)$ & $13.059(0.015)$ & $12.863(0.017)$ & 1 \\
\hline 505.72 & $13.179(0.015)$ & $12.977(0.015)$ & $12.823(0.021)$ & 1 \\
\hline 507.80 & $13.158(0.015)$ & $13.041(0.029)$ & $12.812(0.031)$ & 1 \\
\hline 510.75 & $13.155(0.015)$ & $12.998(0.015)$ & $12.845(0.039)$ & 1 \\
\hline 513.72 & $13.089(0.016)$ & $12.959(0.030)$ & $12.733(0.027)$ & 1 \\
\hline 516.71 & $12.885(0.015)$ & $12.811(0.015)$ & $12.665(0.015)$ & 1 \\
\hline 519.72 & $12.981(0.015)$ & $12.844(0.017)$ & $12.535(0.015)$ & 1 \\
\hline 522.59 & $12.997(0.015)$ & $12.779(0.015)$ & $12.658(0.021)$ & 1 \\
\hline 527.63 & $12.942(0.015)$ & $12.770(0.015)$ & $12.523(0.016)$ & 1 \\
\hline 528.59 & $12.947(0.015)$ & $12.754(0.015)$ & $12.544(0.017)$ & 1 \\
\hline 538.60 & $12.894(0.015)$ & $12.710(0.015)$ & $12.412(0.015)$ & 1 \\
\hline 546.61 & $12.875(0.015)$ & $12.669(0.015)$ & $12.514(0.025)$ & 1 \\
\hline 547.60 & $12.843(0.015)$ & $12.687(0.015)$ & $12.503(0.017)$ & 1 \\
\hline 551.66 & $12.917(0.015)$ & $12.716(0.015)$ & $12.552(0.015)$ & 1 \\
\hline 558.55 & $12.828(0.015)$ & $12.730(0.015)$ & $12.469(0.015)$ & 1 \\
\hline 565.57 & $12.971(0.015)$ & $12.721(0.015)$ & $12.538(0.015)$ & 1 \\
\hline 572.54 & $12.944(0.015)$ & $12.842(0.015)$ & $12.656(0.038)$ & 1 \\
\hline 578.55 & $13.153(0.015)$ & $13.028(0.015)$ & $12.854(0.015)$ & 1 \\
\hline 586.52 & & $13.163(0.015)$ & $\ldots$ & 1 \\
\hline 592.53 & $13.573(0.015)$ & $13.376(0.035)$ & $13.095(0.039)$ & 1 \\
\hline 599.52 & $13.992(0.047)$ & $13.633(0.039)$ & $13.531(0.038)$ & 1 \\
\hline 606.54 & $14.568(0.040)$ & $14.198(0.043)$ & $14.041(0.047)$ & 1 \\
\hline 613.53 & $14.619(0.057)$ & $14.341(0.053)$ & $14.186(0.064)$ & 1 \\
\hline 620.52 & $14.815(0.042)$ & $14.433(0.047)$ & $14.151(0.044)$ & 1 \\
\hline 627.50 & $14.778(0.034)$ & $14.513(0.045)$ & $14.271(0.029)$ & 1 \\
\hline 634.49 & $14.922(0.038)$ & $14.618(0.051)$ & $14.400(0.038)$ & 1 \\
\hline 638.51 & $14.886(0.045)$ & $\cdots$ & $\cdots$ & 2 \\
\hline 639.49 & $14.860(0.055)$ & $14.585(0.036)$ & $\ldots$ & 2 \\
\hline 640.49 & $14.898(0.057)$ & $14.603(0.041)$ & $\cdots$ & 2 \\
\hline 641.48 & $14.888(0.059)$ & & $14.521(0.087)$ & 2 \\
\hline 641.50 & $14.881(0.023)$ & $14.677(0.035)$ & $14.384(0.033)$ & 1 \\
\hline 642.48 & $\cdots$ & $14.651(0.034)$ & $14.568(0.084)$ & 2 \\
\hline 643.49 & $14.934(0.041)$ & & $14.607(0.050)$ & 2 \\
\hline 644.48 & $14.938(0.056)$ & $14.656(0.037)$ & $\ldots$ & 2 \\
\hline 650.48 & $15.017(0.048)$ & $\cdots$ & $\cdots$ & 2 \\
\hline 653.48 & $15.064(0.019)$ & $14.908(0.028)$ & $14.654(0.038)$ & 1 \\
\hline 655.48 & $15.098(0.047)$ & $14.958(0.023)$ & $14.779(0.029)$ & 1 \\
\hline 656.48 & $15.200(0.020)$ & $15.015(0.021)$ & $14.818(0.033)$ & 1 \\
\hline 670.46 & $15.384(0.020)$ & $\ldots$ & $\ldots$ & 1 \\
\hline
\end{tabular}

Notes.

a Julian Date minus 2,451,000.

b 1 = CTIO $1.0 \mathrm{~m}$ (YALO) using $J H K$ filters; $2=$ LCO $1.0 \mathrm{~m}$ using $J_{s} H K_{s}$ filters.

an accurate position of SN 2003hn to be R.A. = 3:44:36.27, decl. $=-44: 37: 50.1$ (J2000).

A finder chart is shown in Figure 2. A spectrum obtained with the $2.3 \mathrm{~m}$ telescope of the Australian National University on 26.7 August UT by Salvo et al. (2003) indicated that SN 2003hn was a Type II-P SN approximately two weeks after explosion. 

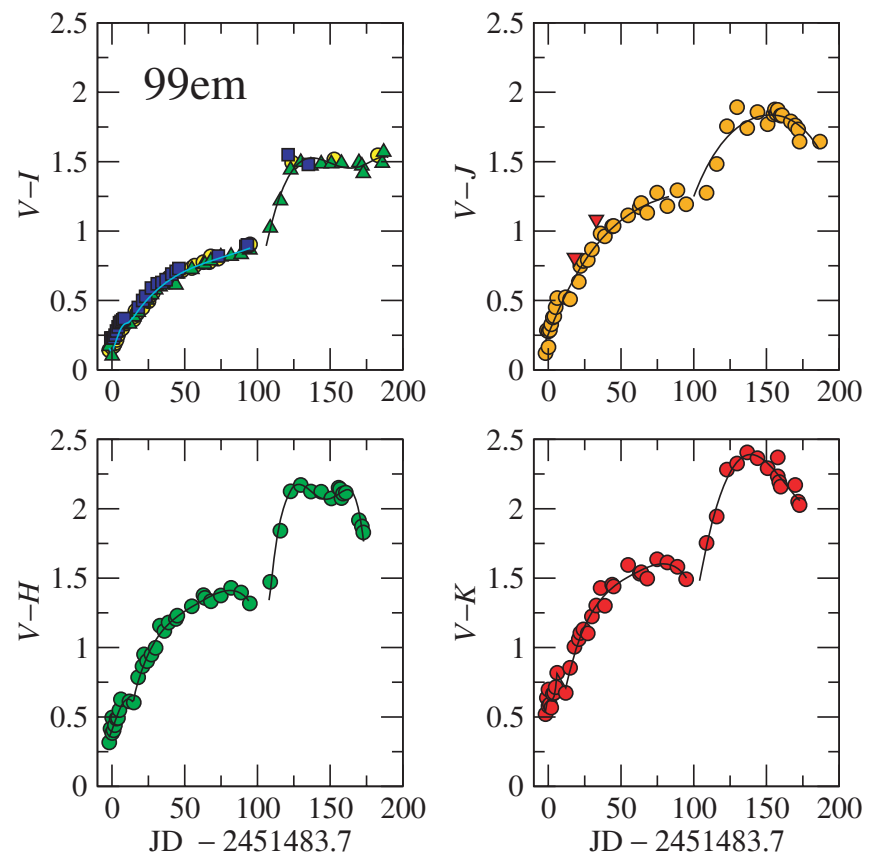

Figure 1. Optical/IR colors of SN 1999em vs. number of days since reference time, JD 2,451,483.7. In addition to the near-IR data presented in this paper, we have used optical data of Leonard et al. (2002) and M. Hamuy et al. (2008, in preparation). The $V-R$ template of SN 1999em excludes the $R$ band taken with ANDICAM. At that time, ANDICAM had a very broad, nonstandard $R$-band filter. We have fit lower-order polynomials to sections of the data. Two points in the upper-right diagram, marked by the triangles, have been treated as outliers and were excluded from the fits.

(A color version of this figure is available in the online journal.)

For the calibration of the optical photometry of SN 2003hn we adopt the magnitudes and colors of the NGC 1448 field stars previously given by us (Krisciunas et al. 2003). From further observations of this field on photometric nights in 2003 using Landolt (1992) standards we uncovered no anomalies in the optical calibration. However, our previous IR calibration of one of the field stars is wrong. The star in question is labeled "C2" in Figure 2. Krisciunas et al. (2003) refer to it as "star 7" of their photometric sequence. From new observations on four photometric nights using infrared standards of Persson et al. (1998) we obtain the following photometry for "star 7": $J=12.964 \pm 0.007, H=12.500 \pm 0.009$, and $K=12.395 \pm$ 0.025 . These can be compared to the values from 2MASS of $J=12.916 \pm 0.021, H=12.478 \pm 0.021$, and $K=12.439 \pm$ 0.027 . Our JHK photometry of "star 6" (= "C3" in Figure 2) obtained in 2003 is within a few thousandths of a magnitude of the values previously published.

ANDICAM also contains a $1.03 \mu \mathrm{m}$ filter known as $Y$ (Hillenbrand et al. 2002). From synthetic photometry of Kurucz model spectra spanning a range of temperatures Hamuy et al. (2006, Appendix C) obtained a relationship between $Y-K_{s}$ colors and the published $J-K_{s}$ colors of Persson et al. (1998) standards. For "star 6" of the NGC 1448 sequence we adopt $Y=14.102 \pm 0.010$, and for "star 7" $Y=13.279 \pm 0.018$.

Our optical photometry of SN 2003hn was derived using sharp reference templates obtained with the CTIO $0.9 \mathrm{~m}$ telescope on 2002 February 19 (UT), long before the SN exploded. The Las Campanas optical data (M. Hamuy et al. 2008, in preparation) used templates obtained on 2004 October 4 and 2004 November 11. It is possible that some of their late-time photometry is affected by the (faint) presence of the $\mathrm{SN}$ in their templates.

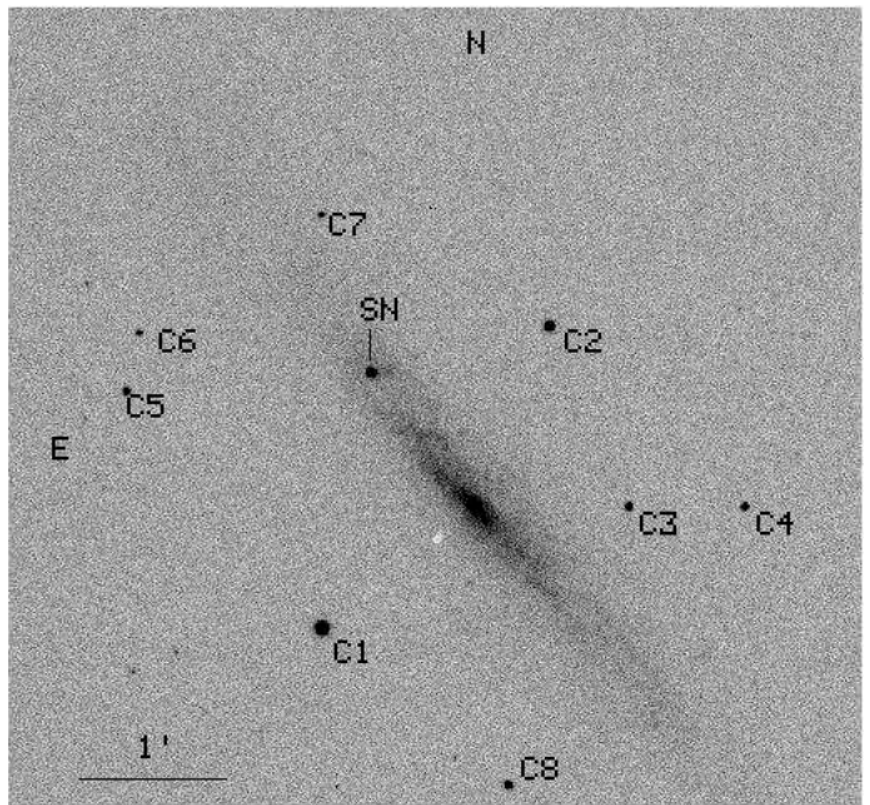

Figure 2. The finder chart for NGC 1448 and SN 2003hn. This is a $V$-band image obtained with the Las Campanas $1.0 \mathrm{~m}$ telescope on 2003 August 28 UT. The stars "C2" and "C3" here are the same as "star 7" and "star 6," respectively, of the photometric sequence of Krisciunas et al. (2003).

For our optical photometry we used image subtraction scripts provided to us by Brian Schmidt, which rely on the point-spread function (PSF) matching technique of Alard \& Lupton (1998). This provided us with PSF magnitudes of the $\mathrm{SN}$ and the field stars. We then completed the data reduction in the IRAF environment using the photcal package.

In Table 2, we present UBVRI photometry of SN 2003hn obtained at CTIO. Near-IR photometry obtained with the CTIO $1.3 \mathrm{~m}$ telescope is given in Table 3 .

Filter-by-filter photometry of SN 2003hn is shown in Figure 3. Photometric colors based solely on optical data of SN 2003hn are shown in Figure 4. V minus IR colors of SN 2003hn are shown in Figure 5. While the plateau phase of the light curves of SN 1999em lasted roughly for 100 days, the plateau phase of SN 2003hn lasted about 20 days less. The onset of the sudden rise in many color indices at late times occurred about 20 days earlier in SN 2003hn.

M. Jones et al. (2008, in preparation) have carried out an EPM analysis of the spectra of SN 2003hn obtained at Las Campanas Observatory (LCO). Their derived explosion time is JD 2,452,857 \pm 4 days. The large uncertainty in the date of explosion is due to the lack of early-time spectra compared to SN $1999 \mathrm{em}$. The method of F. Olivares et al. (2008, in preparation) relies on the end of the plateau phase as the reference time. This is not the best reference time to choose for our purposes here, as the plateau phase of SN 2003hn lasted 20 days less than that of SN $1999 \mathrm{em}$. We choose to adopt a reference time for SN 2003hn which relies on a $\chi^{2}$ minimization of the color curves using the templates from SN $1999 \mathrm{em}$ for fitting. The most consistent results were obtained using the $V-J, V-H$, and $V-K$ templates and adding $1386.78 \pm 0.45$ days to the reference time adopted for SN 1999em. Thus, for SN $2003 \mathrm{hn}$ we obtain a reference time of JD 2,452,870.48 \pm 0.45 .

If two SNe have identical spectra but suffer different amounts of total extinction along the line of sight, all color indices of one object should be redder than the corresponding color 
Table 2

Optical Photometry of SN 2003hn

\begin{tabular}{lcccccc}
\hline \hline $\mathrm{JD}^{\mathrm{a}}$ & $U$ & $B$ & $V$ & $R$ & $I$ & Telescope $^{\mathrm{b}}$ \\
\hline 2877.82 & $13.941(0.011)$ & $14.642(0.031)$ & $14.464(0.038)$ & $14.163(0.031)$ & $14.078(0.036)$ & 1 \\
2878.79 & $13.953(0.041)$ & $14.603(0.026)$ & $14.435(0.026)$ & $14.175(0.012)$ & $14.046(0.026)$ & 2 \\
2879.80 & $14.079(0.010)$ & $14.703(0.037)$ & $14.499(0.043)$ & $14.163(0.037)$ & $14.086(0.037)$ & 1 \\
2883.82 & $14.543(0.018)$ & $14.857(0.037)$ & $14.468(0.033)$ & $14.143(0.037)$ & $14.060(0.034)$ & 1 \\
2891.81 & $15.619(0.032)$ & $15.323(0.031)$ & $14.637(0.038)$ & $14.231(0.037)$ & $14.077(0.024)$ & 1 \\
2899.82 & $16.611(0.038)$ & $15.692(0.032)$ & $14.805(0.036)$ & $14.373(0.038)$ & $14.186(0.042)$ & 1 \\
2905.85 & $16.797(0.045)$ & $15.906(0.026)$ & $14.856(0.029)$ & $14.407(0.032)$ & $14.222(0.033)$ & 1 \\
2905.88 & $\ldots$ & $15.881(0.018)$ & $14.903(0.014)$ & $14.448(0.015)$ & $14.185(0.023)$ & 2 \\
2911.80 & $17.099(0.063)$ & $16.137(0.029)$ & $14.978(0.041)$ & $14.465(0.033)$ & $14.223(0.028)$ & 1 \\
2918.78 & $17.262(0.071)$ & $16.256(0.025)$ & $15.043(0.034)$ & $14.547(0.033)$ & $14.279(0.032)$ & 1 \\
2925.75 & $17.620(0.103)$ & $16.415(0.034)$ & $15.116(0.038)$ & $14.606(0.042)$ & $14.306(0.030)$ & 1 \\
2933.73 & $17.984(0.143)$ & $16.563(0.031)$ & $15.192(0.040)$ & $14.631(0.029)$ & $14.374(0.042)$ & 1 \\
2939.86 & $18.204(0.037)$ & $16.663(0.021)$ & $15.282(0.019)$ & $14.702(0.017)$ & $14.375(0.016)$ & 2 \\
2940.75 & $18.476(0.235)$ & $16.744(0.034)$ & $15.249(0.038)$ & $14.722(0.039)$ & $14.402(0.029)$ & 1 \\
2947.68 & $\ldots$ & $16.917(0.039)$ & $15.400(0.035)$ & $14.786(0.040)$ & $14.527(0.043)$ & 1 \\
2954.69 & $\ldots$ & $17.210(0.027)$ & $15.581(0.032)$ & $14.950(0.039)$ & $14.614(0.031)$ & 1 \\
2962.66 & $\ldots$ & $17.990(0.039)$ & $16.220(0.034)$ & $15.440(0.029)$ & $15.098(0.049)$ & 1 \\
2967.75 & $20.681(0.171)$ & $18.881(0.025)$ & $17.134(0.020)$ & $16.208(0.015)$ & $15.756(0.017)$ & 2 \\
2969.65 & $\ldots$ & $19.219(0.200)$ & $17.405(0.100)$ & $16.364(0.035)$ & $15.964(0.036)$ & 1 \\
2990.63 & $\ldots$ & $19.571(0.080)$ & $17.749(0.060)$ & $16.678(0.028)$ & $16.299(0.035)$ & 1 \\
2997.69 & $\ldots$ & $19.611(0.080)$ & $17.840(0.060)$ & $16.811(0.035)$ & $16.398(0.035)$ & 1 \\
3030.66 & $\ldots$ & $19.896(0.045)$ & $18.202(0.030)$ & $17.193(0.022)$ & $\ldots$ & 2 \\
\hline & & & & &
\end{tabular}

Notes.

a Julian Date minus 2,450,000.

b $1=$ CTIO $1.3 \mathrm{~m} ; 2=$ CTIO $0.9 \mathrm{~m}$.

Table 3

Near-IR Photometry of SN 2003hn

\begin{tabular}{lcccc}
\hline \hline $\mathrm{JD}^{\mathrm{a}}$ & $Y$ & $J$ & $H$ & $K$ \\
\hline 2877.82 & $13.976(0.031)$ & $13.886(0.010)$ & $13.670(0.017)$ & $\ldots$ \\
2879.80 & $13.987(0.020)$ & $13.820(0.010)$ & $13.663(0.011)$ & $13.481(0.030)$ \\
2883.82 & $13.914(0.022)$ & $13.739(0.013)$ & $13.570(0.014)$ & $13.333(0.033)$ \\
2891.81 & $13.884(0.020)$ & $13.687(0.010)$ & $13.531(0.011)$ & $13.310(0.027)$ \\
2899.79 & $13.949(0.020)$ & $13.724(0.009)$ & $13.557(0.011)$ & $13.318(0.028)$ \\
2905.85 & $13.955(0.020)$ & $13.720(0.009)$ & $13.559(0.011)$ & $13.358(0.027)$ \\
2911.79 & $14.021(0.020)$ & $13.773(0.009)$ & $13.571(0.011)$ & $13.384(0.029)$ \\
2918.77 & $14.062(0.021)$ & $13.790(0.010)$ & $13.589(0.011)$ & $13.274(0.030)$ \\
2925.75 & $14.088(0.020)$ & $13.830(0.009)$ & $13.634(0.011)$ & $13.279(0.032)$ \\
2933.73 & $14.186(0.020)$ & $13.871(0.010)$ & $13.684(0.011)$ & $13.369(0.032)$ \\
2940.75 & $14.198(0.020)$ & $13.914(0.010)$ & $13.706(0.011)$ & $13.414(0.031)$ \\
2947.68 & $14.302(0.021)$ & $14.002(0.010)$ & $13.774(0.012)$ & $13.489(0.030)$ \\
2954.69 & $14.394(0.021)$ & $14.138(0.011)$ & $13.930(0.012)$ & $13.716(0.036)$ \\
2969.65 & $15.707(0.049)$ & $15.231(0.043)$ & $14.787(0.035)$ & $\ldots$ \\
2990.62 & $16.152(0.035)$ & $15.727(0.020)$ & $15.385(0.024)$ & $15.334(0.094)$ \\
2997.69 & $16.262(0.037)$ & $15.845(0.026)$ & $15.540(0.027)$ & $15.505(0.106)$ \\
\hline
\end{tabular}

Note. ${ }^{a}$ Julian Date minus 2,450,000.

indices of the other object. In most color indices $(V-[R I J H K])$ SN 2003hn was redder than SN 1999em. However, until the end of its plateau phase SN 2003hn was bluer than SN 1999em in $U-B$ by $0.154 \mathrm{mag}$ and bluer in $B-V$ by $0.019 \mathrm{mag}$. The simplest explanation is that the spectrum of SN $1999 \mathrm{em}$ exhibits greater line blanketing due to a higher metallicity. Thus, we believe that these two objects do not have identical spectra over the whole optical wavelength range.

In Table 4, we give the magnitude shifts necessary to fit the SN 1999em $V-[R I J H K]$ color templates to the SN 2003hn colors. Under the assumption that the differences are solely due to dust extinction, these color excesses should monotonically increase as the wavelength of the second filter increases. The reddening model of Cardelli et al. (1989) easily allows us to calculate multiplicative factors to scale these color excesses, giving implied values of $\Delta A_{V}$ that should be statistically equal. Column 6 of Table 4 gives these estimates and a weighted mean of $\Delta A_{V}=0.245 \pm 0.025$. This was obtained assuming a value of $R_{V}=3.1$. The small reduced $\chi^{2}$-values indicate that the photometry may actually be more accurate than the formal random errors. If we had used $R_{V}=2.15$, the value derived by Krisciunas et al. (2007) for the host galaxy dust affecting SN $2001 \mathrm{el}$, we would obtain a very similar value of $\Delta A_{V}=0.225 \pm$ 0.023 for the difference of the $V$-band extinctions of SNe 2003hn and $1999 \mathrm{em}$.

In the third column of Table 4, we give the rms scatter of the SN 2003hn data with respect to the adjusted SN 1999em color templates. These values are comparable to the rms scatter 
Table 4

Color Excesses and Implied Values of $\Delta A_{V}{ }^{\mathrm{a}}$

\begin{tabular}{lcccccc}
\hline \hline Color Excess & Value $(\mathrm{mag})$ & rms $(\mathrm{mag})$ & Range $^{\mathrm{b}}$ & $\chi_{v}^{2}$ & Factor $^{\mathrm{c}}$ & $\Delta A_{V}$ \\
\hline$\Delta(V-R)$ & 0.063 & \pm 0.026 & $7-84$ & 0.84 & 4.016 & $0.253(0.104)$ \\
$\Delta(V-I)$ & 0.098 & \pm 0.032 & $7-77$ & 0.58 & 1.919 & $0.188(0.061)$ \\
$\Delta(V-J)$ & 0.184 & \pm 0.033 & $7-84$ & 0.68 & 1.393 & $0.256(0.046)$ \\
$\Delta(V-H)$ & 0.190 & \pm 0.035 & $7-84$ & 0.81 & 1.235 & $0.235(0.043)$ \\
$\Delta(V-K)$ & 0.267 & \pm 0.055 & $7-84$ & 1.33 & 1.129 & $0.301(0.062)$ \\
Weighted Mean & & & & & & $0.245(0.025)$
\end{tabular}

Notes.

${ }^{\text {a }}$ Here we determine the implied amount of extra $V$-band extinction suffered by SN 2003hn compared to SN 1999em.

${ }^{\mathrm{b}}$ Range in days since the reference time of SN 2003hn, JD $=2,452,870.48$.

c Scale factors derived from values of $A_{\lambda} / A_{V}$ in Table 3 of Cardelli et al. (1989), used to obtain $A_{V}$ from color excess. It is assumed that $R_{V}=3.1$.

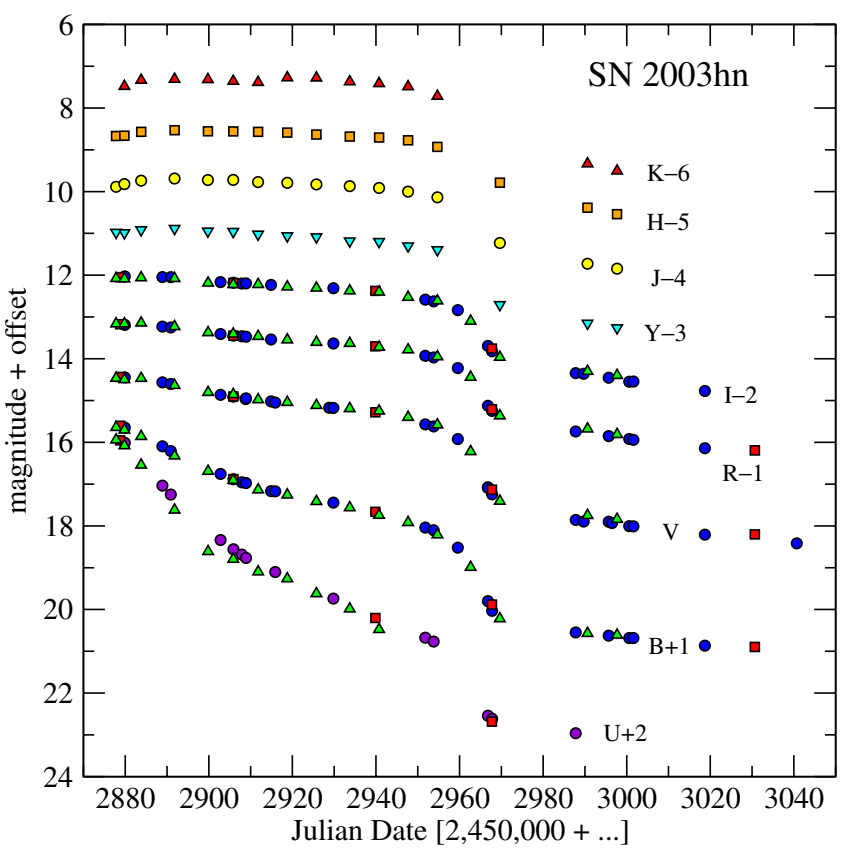

Figure 3. Optical and near-IR photometry of SN 2003hn. The UBVRI data are coded by telescope. Dots $=$ data from LCO $1.0 \mathrm{~m}$; squares $=$ CTIO $0.9 \mathrm{~m}$; triangles $=$ CTIO $1.3 \mathrm{~m}$. All the infrared data were obtained with the CTIO $1.3 \mathrm{~m}$. Except for some of the late-time $U$ - and $B$-band data, the uncertainties of the data points are comparable to, or smaller than, the size of the symbols.

(A color version of this figure is available in the online journal.)

of the SN 1999em data used to create the templates. Thus, the shape and precision of the two sets of color curves are comparable.

Baron et al. (2000) used the spectral synthesis code SYNOW to fit ground-based spectra and one Hubble Space Telescope (HST) spectrum of SN 1999em obtained within a week of its discovery. To match the observed spectra with SYNOW model spectra required reddening the latter by $E(B-V)=0.10$, with an uncertainty of \pm 0.05 mag. Assuming "standard" $R_{V}=3.1$ dust implies that the total $V$-band extinction suffered by $\mathrm{SN}$ $1999 \mathrm{em}$ was $A_{V}=0.31 \pm 0.15 \mathrm{mag}$. F. Olivares et al. (2008, in preparation) find $A_{V}($ host $)=0.24 \pm 0.14$, implying $A_{V}$ (total $)=$ 0.364 for SN $1999 \mathrm{em}$. We shall adopt $A_{V}$ (total) $=0.34 \pm 0.14$ for SN 1999em.

The implication is that the line of sight to SN 2003hn was affected by a total extinction of $A_{V}=0.58 \pm 0.14$ mag. Only $0.043 \mathrm{mag}$ of this extinction is due to dust in our Galaxy,
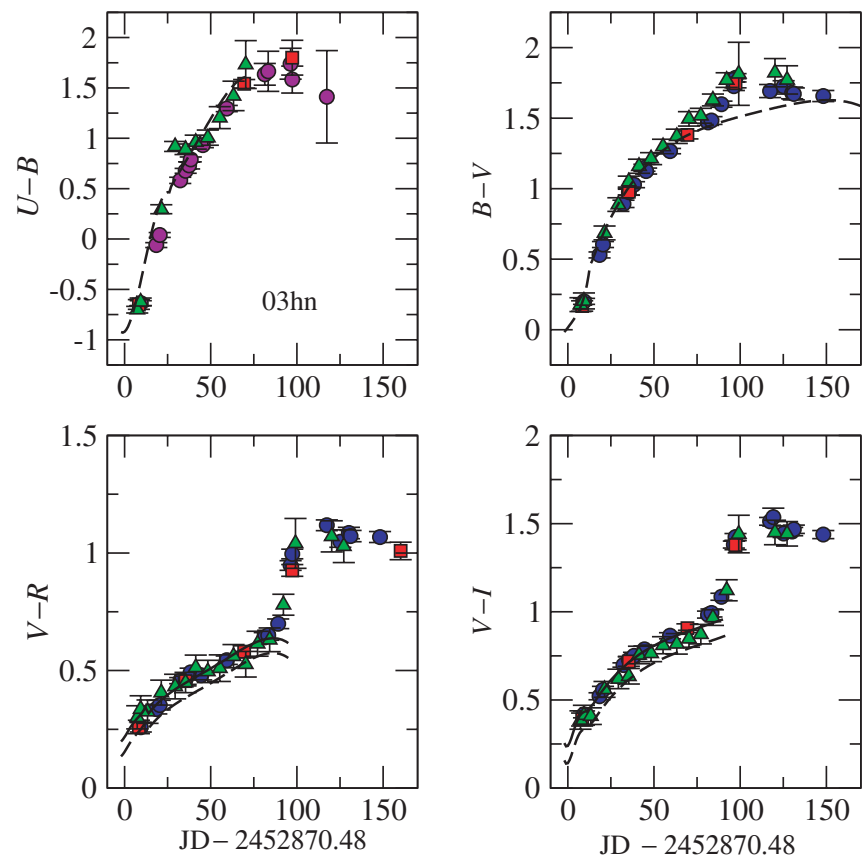

Figure 4. Photometric colors of SN 2003 hn based solely on optical photometry. The abscissa represents the number of days since the reference time JD 2,452,870.48. The dashed curves are the loci from SN 1999em, using data from Leonard et al. (2002) and M. Hamuy et al. (2008, in preparation). The solid lines for $V-R$ and $V-I$ are the loci from the SN 1999em offset by amounts that minimize the reduced $\chi^{2}$ of the fits.

(A color version of this figure is available in the online journal.)

as $E(B-V)_{\mathrm{Gal}}=0.014$ toward NGC 1448 (Schlegel et al. 1998). F. Olivares et al. (2008, in preparation) obtain $A_{V}$ (total) $=0.50 \pm 0.14 \mathrm{mag}$ from a consideration of the $V-I$ color only. At our request, L. Dessart (2008, in preparation) kindly performed fits of Type II-P SN atmosphere models to our optical spectra of SN 2003hn. In these fits the spectral lines are used to constrain the photospheric temperature and the corresponding continuum is employed to estimate the extinction. His analysis yields $A_{V}$ (total) $=0.63 \pm 0.25 \mathrm{mag}$. These three estimates are remarkably consistent. It is more likely that our optical/ IR derived value is closest to the truth, as it relies on a large number of photometric bands extending over a wider range of wavelengths than either of the other two methods. Also, since interstellar extinction is less problematic at IR wavelengths, $V$ minus IR color excesses asymptotically approach $A_{V}$ as the 

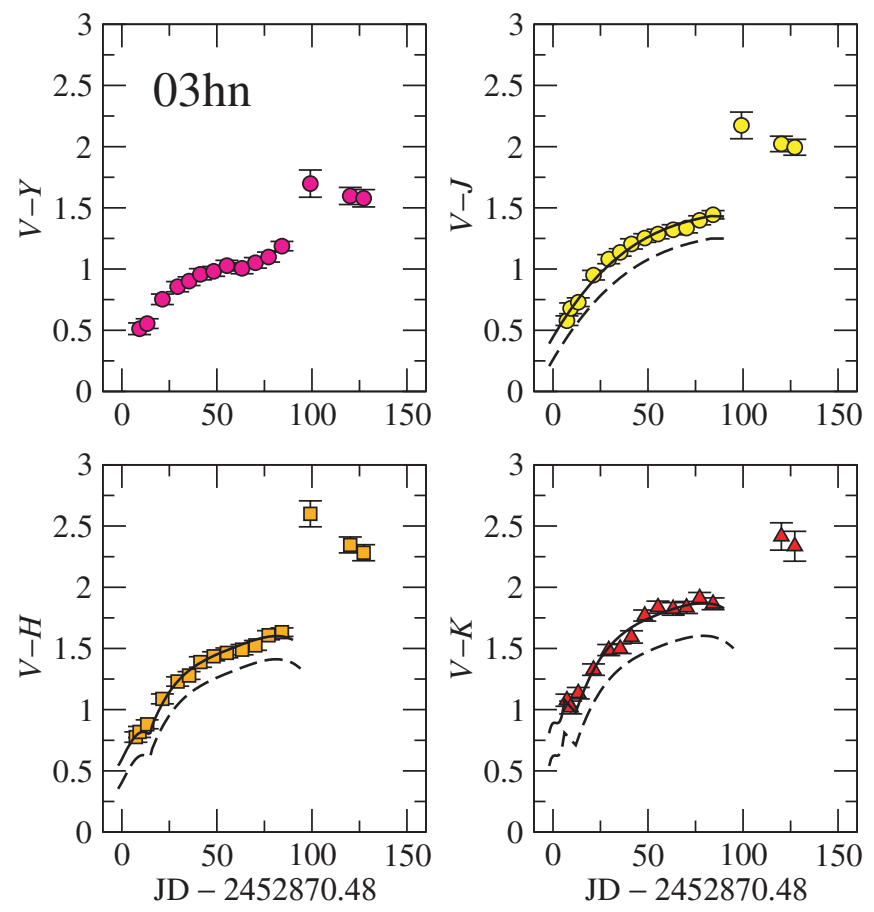

Figure 5. $V$ minus near-IR colors of SN 2003 hn vs. days since the reference time JD 2,452,870.48. As in Figure 4, the dashed lines are the loci from SN $1999 \mathrm{em}$, and the solid lines are those loci offset to minimize the reduce $\chi^{2}$ of the fits to the data of SN $2003 \mathrm{hn}$.

(A color version of this figure is available in the online journal.)

wavelength of the IR band under consideration becomes longer and longer.

In Table 5, we summarize the available spectra of SN 2003hn. The first spectrum was obtained with the $2.3 \mathrm{~m}$ telescope of the Australian National University and the remaining nine spectra at LCO. This spectroscopic time series, shown in Figure 6, starts 21 days after explosion (assuming the time of explosion on JD $2452857 \pm 4$ derived by M. Jones et al. 2008, in preparation, using the EPM) and covers 163 days of evolution of SN $2003 \mathrm{hn}$. The strongest $\mathrm{SN}$ lines are indicated along with the telluric lines (by the letter " $T$ "). The first spectrum shows a blue continuum, P-Cygni profiles for the $\mathrm{H}$ Balmer lines, and a weak He I $\lambda 5876$ line which is characteristic of Type II SNe during their initial hottest phases. The expansion velocity from the minimum of the $\mathrm{H} \beta$ absorption feature was $\sim 9400 \mathrm{~km} \mathrm{~s}^{-1}$, which is typical of Type II SNe during the initial phases. The presence of the interstellar Na I D lines $\lambda \lambda 5890,5896$ with an equivalent width of $0.8 \pm 0.1 \AA$ suggest nonnegligible interstellar absorption in the host galaxy (in agreement with our analysis of the SN colors shown above). As the $\mathrm{SN}$ evolved, the atmospheric temperature dropped, the He I $\lambda 5876$ line disappeared, and several new lines became evident, namely, the Ca II H\&K $\lambda \lambda 3934,3968$ blend, the Ca II triplet $\lambda \lambda 8498,8542,8662$, the Na I D blend, and several lines attributed to Fe II (see Jeffery \& Branch 1990 for a list of line identifications). By day 90 , the color temperature was only $5000 \mathrm{~K}$, approximately the recombination temperature of $\mathrm{H}$. This spectrum corresponds to the end of plateau or the optically thick phase. The last three spectra were taken during the nebular phase as can be appreciated from the relatively fainter continuum and the increasingly weaker absorption features.

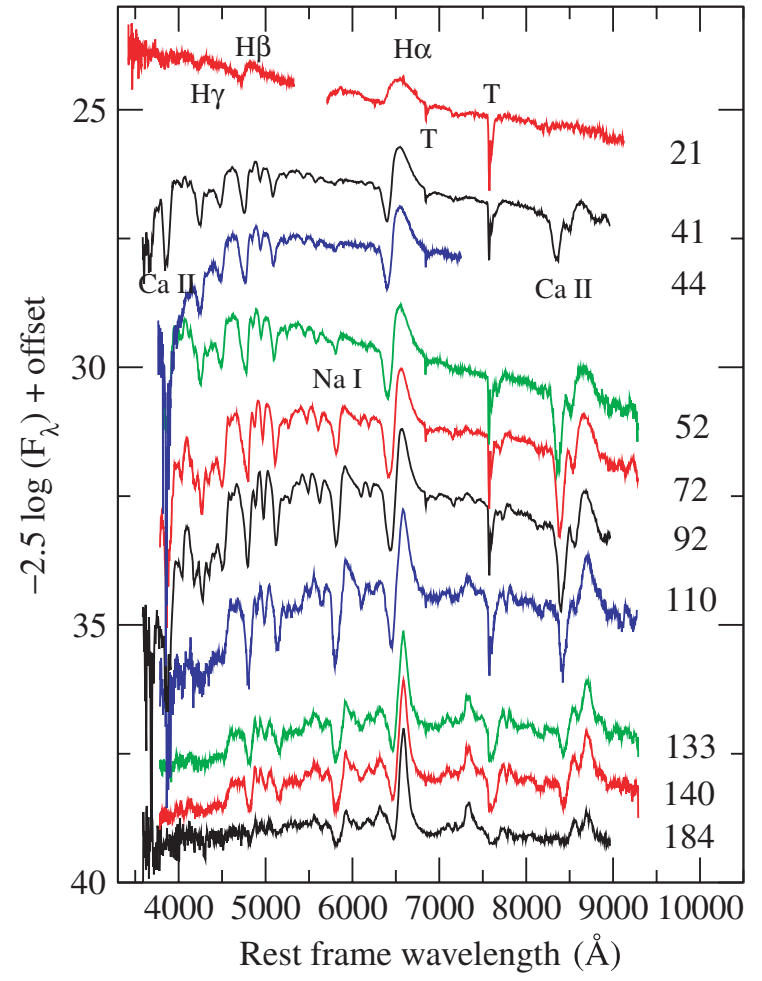

Figure 6. Spectra of SN 2003hn. See Table 5 for further details. The numbers within the graph are the number of days since the time of explosion, JD 2,452,857 \pm 4 (M. Jones et al. 2008, in preparation). The features labeled "T" are terrestrial features due to the Earth's atmosphere.

(A color version of this figure is available in the online journal.)

\section{DISCUSSION}

Hamuy et al. (2001) give an EPM distance to SN 1999em of $7.5 \pm 0.5 \mathrm{Mpc}$. A similar analysis by Leonard et al. (2002) yields $8.2 \pm 0.6 \mathrm{Mpc}$. Using improved photospheric velocities, M. Jones et al. (2008, in preparation) obtain a distance of $9.3 \pm$ $0.5 \mathrm{Mpc}$. All these EPM distances were obtained using the same set of dilution factors calculated by Eastman et al. (1996). Recently a new set of atmosphere models was calculated by Dessart \& Hillier (2005) whose dilution factors are systematically higher than those of Eastman et al. and which lead to greater distances. Based on these new models Dessart \& Hillier (2006) and M. Jones et al. (2008, in preparation) obtain $11.5 \pm 1.0$ and $13.9 \pm 1.4 \mathrm{Mpc}$, respectively. Leonard et al. (2003) give a Cepheid-based distance of $11.7 \pm 1.0 \mathrm{Mpc}$, in good agreement with the EPM results derived from the new models of Dessart \& Hillier (2005), which are considerably greater than those obtained with the Eastman et al. (1996) models. If we adopt the Cepheid-based distance-the maximum observed $V$-band magnitude of 13.79 and a total $V$-band extinction of $0.34 \mathrm{mag}$-it follows that $M_{V}=-16.89 \pm 0.24 \mathrm{mag}$ for SN $1999 \mathrm{em}$.

Using the $\Delta m_{15}(B)$ method of Phillips et al. (1999), the distance to NGC 1448, the host of SNe 2001el and 2003hn, is $17.9 \pm 0.8 \mathrm{Mpc}$ (Krisciunas et al. 2003). Adopting the total $V$-band extinction of $0.586 \pm 0.050 \mathrm{mag}$ for SN 2001el (Krisciunas et al. 2007), it follows that $M_{V}(\max )=-19.12$ \pm 0.11 . A check on the distance of NGC 1448 can be obtained by assuming that the $J H K$ absolute magnitudes at maximum of SN 2001el equal the mean values given in Table 17 of Krisciunas et al. (2004b). Krisciunas et al. (2007) also found that $R_{V}=2.15$ is the most appropriate value for the host galaxy dust associated 
Table 5

$\log$ of SN 2003hn Spectra

\begin{tabular}{lcccccc}
\hline \hline JD $^{\mathrm{a}}$ & UT Date & $\Delta T^{\mathrm{b}}$ & Telescope $^{\mathrm{c}}$ & $\lambda$ Range $(\AA)$ & $\Delta \lambda(\AA)$ & Exposure Time $(\mathrm{s})$ \\
\hline 2878.20 & 2003 Aug 26 & 21 & 1 & $3433-9162$ & $1.1 / 2.1$ & $600 \times 2$ \\
2897.86 & 2003 Sep 15 & 41 & 2 & $3600-9000$ & 4.2 & 90 \\
2900.87 & 2003 Sep 18 & 44 & 3 & $3780-7280$ & 2.0 & 300 \\
2908.83 & 2003 Sep 26 & 52 & 3 & $3800-9325$ & 3.1 & 600 \\
2928.83 & 2003 Oct 16 & 72 & 3 & $3800-9325$ & 3.1 & 600 \\
2948.83 & 2003 Nov 05 & 92 & 2 & $3600-9000$ & 4.2 & 150 \\
2966.81 & 2003 Nov 23 & 110 & 3 & $3800-9325$ & 3.1 & 600 \\
2989.75 & 2003 Dec 16 & 133 & 3 & $3800-9325$ & 3.1 & 600 \\
2996.68 & 2003 Dec 23 & 140 & 3 & $3800-9325$ & 3.1 & 600 \\
3040.69 & 2004 Feb 05 & 184 & 2 & $3600-9000$ & 4.2 & 600 \\
\hline
\end{tabular}

Notes.

a Julian Date minus 2,450,000.

b Number of days since the derived explosion time of JD 2,452,857 \pm 4 (M. Jones et al. 2008, in preparation).

${ }^{c} 1=$ Australian National University $2.3 \mathrm{~m} ; 2=$ Magellan \#2 (6.5 m Clay Telescope); $3=2.5 \mathrm{~m}$ DuPont Telescope.

with SN 2001el. From the near-IR maxima we obtain a distance of $18.1 \pm 0.4 \mathrm{Mpc}$. For comparison, the EPM analysis of SN $2003 \mathrm{hn}$ by M. Jones et al. (2008, in preparation) yields $16.9 \pm 2$ and $26.3 \pm 7 \mathrm{Mpc}$, using dilution factors from Eastman et al. (1996) and Dessart \& Hillier (2005), respectively. The Standardized Candle Method (SCM) applied to SN 2003hn yields a distance of $17.8 \pm 1 \mathrm{Mpc}$ (F. Olivares et al. 2008, in preparation).

Under the assumption that the early-time photometric behavior of SN 2003hn was the same as that of SN 1999em, we can extrapolate that SN 2003hn was 0.056 mag brighter than our earliest $V$-band measurement. Adopting $V_{\max }=14.41 \pm$ $0.03, A_{V}($ total $)=0.58 \pm 0.14$, and $d=17.9 \pm 0.8 \mathrm{Mpc}$, it follows that $M_{V}(\max )=-17.44 \pm 0.17$. Taken at face value, SN 2003hn was $0.55 \pm 0.30$ mag brighter than its "cousin" SN 1999em. Since SN 2003hn and SN 2001el occurred in the same galaxy, a comparison of their absolute magnitude differences involves no uncertainties in distance. Corrected for extinction, at the time of maximum light SN 2003hn was 1.68 mag fainter in $V$ than SN 2001el. This confirms the notion that a typical Type II-P SN is significantly fainter than a Type Ia SN for the optical maxima.

In the near-IR, SNe 2001el and 2003hn are not so dissimilar in brightness at maximum. For SN 2001el the observed $K_{\max }=$ $12.83 \pm 0.04$ (Krisciunas et al. 2003), its total $K$-band extinction was about $0.057 \mathrm{mag}$, and absolute magnitude $M_{K}(\max ) \approx$ -18.49 . For SN 2003hn, $K_{\max }=13.27 \pm 0.03$ (from Table 3), $A_{K} \approx 0.064 \mathrm{mag}$, so $M_{K}(\max ) \approx-18.06$. This is only $0.43 \mathrm{mag}$ fainter than SN 2001el. The implication is that a wide angle SN survey carried out at $2.2 \mu \mathrm{m}$ would find Type II-P SNe almost as easily as Type Ia SNe.

Since Type II SNe are single massive stars that have very short main-sequence lifetimes, they end their lives very close to where they were born-in regions of significant levels of star formation. As a result, the light curves of most (or all) Type II $\mathrm{SNe}$ would be affected by interstellar extinction along the line of sight.

We have found two Type II-P SNe whose color curves vary in a similar manner until the end of the plateau phase. The similarities of these color curves and increasing color excesses as we proceed from $V-R$ through $V-K$, imply that the observed color differences are simply due to differing amounts of dust along the line of sight. The implication is that some fraction of Type II-P SNe may exhibit sufficiently uniform color curves that they may be used for a determination of the relative amounts of dust extinction that they suffer. Once we are confident we have data on Type II-P SNe which are minimally reddened in their host galaxies, we can obtain accurate total extinctions for all the SNe with good optical and IR light curves, which show those similar color curves. This will lead to a more accurate distance calibration for Type II-P SNe. As future projects such as Pan-STARRS (Panoramic Survey Telescope and Rapid Response System) and LSST (Large Synoptic Survey Telescope) discover large numbers of $\mathrm{SNe}$, we should be able to use Type Ia and Type II-P SNe for cosmology.

The CTIO $1.3 \mathrm{~m}$ telescope is operated by the Small and Moderate Aperture Research Telescope System (SMARTS) Consortium. We are particularly grateful for the scheduling flexibility of SMARTS. ANDICAM was designed by Darren Depoy of Ohio State University. Without the flexibility of the operation of the $1.3 \mathrm{~m}$ telescope and the dual channel design of ANDICAM, this paper could not have been written. We used data from the 2MASS. We thank Brian Schmidt for providing the classification spectrum of SN 2003hn. M.H. acknowledges support provided by NASA through Hubble Fellowship grant HST-HF-01139.01-A, by Fondecyt through grant 1060808, Centro de Astrofísica FONDAP 15010003, the Millennium Center for Supernova Science through grant P06-045-F funded by "Programa Bicentenario de Ciencia y Tecnología de CONICYT" and "Programa Iniciativa Científica Milenio de MIDEPLAN."

\section{REFERENCES}

Alard, C., \& Lupton, R. H. 1998, ApJ, 503, 325

Baron, E., et al. 2000, ApJ, 545, 444

Cardelli, J. A., Clayton, G. C., \& Mathis, J. S. 1989, ApJ, 345, 245

Dessart, L., \& Hillier, D. J. 2005, A\&A, 439, 671

Dessart, L., \& Hillier, D. J. 2006, A\&A, 447, 691

Eastman, R. G., Schmidt, B. P., \& Kirshner, R. 1996, ApJ, 466, 911

Evans, R. 2003, IAU Circ. 8186

Hamuy, M. 2001, PhD thesis, Univ. of Arizona

Hamuy, M. 2003, ApJ, 582, 905

Hamuy, M., Phillips, M. M., Suntzeff, N. B., Schommer, R. A., Maza, J., Smith, R. C., Lira, P., \& Avilés, R. 1996, AJ, 112, 2438

Hamuy, M., \& Pinto, P. A. 2002, ApJ, 566, 63

Hamuy, M., et al. 2001, ApJ, 558, 615

Hamuy, M., et al. 2006, PASP, 118, 2

Heger, A., Fryer, C. L., Woosley, S. E., Langer, N., \& Hartmann, D. H. 2003 , ApJ, 591, 288

Hendry, M. A., et al. 2006, MNRAS, 369, 1303 
Hillenbrand, L. A., Foster, J. B., Persson, S. E., \& Matthews, K. 2002, PASP, 114,708

Jeffery, D. J., \& Branch, D. 1990, in Jerusalem Winter School for Theoretical Physics. Supernovae, Vol. 6, ed. J. C. Wheeler, T. Piran, \& S. Weinberg (Singapore: World Scientific), 149

Jha, S., Challis, P., Garnavich, P., \& Kirshner, R. 1999, IAU Circ. 7296

Jha, S., Riess, A. G., \& Kirshner, R. P. 2007, ApJ, 659, 122

Krisciunas, K., \& Espinoza, J. 1999, IAU Circ. 8186

Krisciunas, K., Phillips, M. M., \& Suntzeff, N. B. 2004a, ApJ, 602, L81

Krisciunas, K., et al. 2003, AJ, 125, 166

Krisciunas, K., et al. 2004b, AJ, 128, 3034

Krisciunas, K., et al. 2007, AJ, 133, 58

Landolt, A. U. 1992, AJ, 104, 340

Leonard, D. C., Kanbur, S. M., Ngeow, C. C., \& Tanvir, N. R. 2003, ApJ, 594, 247

Leonard, D. C., et al. 2002, PASP, 114, 35

Li, W. D. 1999, IAU Circ. 7294
Li, W. D., Wang, X., Van Dyk, S. D., Cuillandre, J-C., Foley, R. J., \& Filippenko, A. V. 2007, ApJ, 661, 1013

Litvinova, I. I., \& Nadezhin, D. K. 1983, Ap\&SS, 89, 89

Livio, M. 2000, in The Greatest Explosions since the Big Bang: Supernovae and Gamma-Ray Bursts, ed. M. Livio, N. Panagia, \& K. Sahu (Baltimore, MD: Space Telescope Science Institute), 334

Perlmutter, S., et al. 1997, ApJ, 483, 565

Persson, S. E., Murphy, D. C., Krzeminski, W., Roth, M., \& Rieke, M. J. 1998, AJ, 116, 2475

Phillips, M. M. 1993, ApJ, 413, L105

Phillips, M. M., Lira, P., Suntzeff, N. B., Schommer, R. A., Hamuy, M., \& Maza, J. 1999, AJ, 118, 1766

Riess, A. G., Press, W. H., \& Kirshner, R. P. 1996, ApJ, 473, 88

Salvo, M., Bessell, M., \& Schmidt, B. 2003, IAU Circ. 8187

Schlegel, D. J., Finkbeiner, D. P., \& Davis, M. 1998, ApJ, 500, 525

Wang, X., et al. 2008, ApJ, 675, 626

Wood-Vasey, W. M., et al. 2007, ApJ, in press, arXiv:0711.2068 